\title{
Correlating Surface Permeability with Intracrystalline Diffusivity in Nanoporous Solids
}

\author{
Lars Heinke ${ }^{1,2,3}$ and Jörg Kärger ${ }^{1}$ \\ ${ }^{1}$ Faculty of Physics and Geosciences, Department of Interface Physics, University of Leipzig, Linnéstraße 5, 04103 Leipzig, Germany \\ ${ }^{2}$ Fritz-Haber-Institute of the Max-Planck-Society, Faradayweg 4-6, 14195 Berlin, Germany \\ ${ }^{3}$ Materials Science Division, Lawrence Berkeley National Laboratory, Berkeley, California 94720, USA
}

(Received 1 July 2010; published 14 February 2011)

\begin{abstract}
The rates of uptake and release of guest molecules in nanoporous solids are often strongly influenced or even controlled by transport resistances at the external surface ("surface barriers") rather than by intraparticle diffusion, which was assumed to be rate controlling in many of the earlier kinetic studies. By correlating the surface resistance with the intracrystalline diffusivity, we develop here a microkinetic model which closely reproduces the experimentally observed results for short-chain alkanes in $\mathrm{Zn}$ (tbip), a member of the novel metal-organic framework family of nanoporous materials. It seems likely that this mechanism, which is shown to provide a rational explanation of the commonly observed discrepancies between "macro" and "micro" measurements of intracrystalline diffusion, may be fairly general.
\end{abstract}

DOI: 10.1103/PhysRevLett.106.074501

PACS numbers: 66.30.Pa, 47.56.+r, 68.43.Jk, 82.75.Jn

Recent progress in the syntheses of nanoporous solids [1-3] has given rise to an impressive array of new structures which are useful for both fundamental research and technological exploitation [4]. In many of the proposed applications the viability is determined by the rates of uptake and release of the guest molecules [5]. These rates depend on essentially two processes: permeation through the surface of the host particle and intraparticle (intracrystalline) guest diffusion. For most host-guest systems, uptake and release rates are influenced (and sometimes controlled) by a finite rate of permeation through the external boundary of the solid adsorbent, i.e., by "surface barriers" [6,7]. Infrared microimaging (IRM) and interference microscopy (IFM) techniques [8-10] provide reliable approaches to the measurement of such resistances, thus providing a sound basis for the development and confirmation of theoretical models to simulate and predict permeation rates through the surface layer of nanoporous materials [11]. In contrast to the situation within the pore space of regularly structured nanoporous materials an "ideal" surface structure cannot be defined without some arbitrariness. Moreover, any given surface structure must be expected to be highly dependent upon the conditions of sample synthesis, storage, pretreatment, and even of the permeation measurements themselves $[12,13]$. Problems with predicting and modeling surface barriers are therefore to be expected whenever the process of permeation through the surface layer depends on a mechanism other than intracrystalline diffusion. Different trends in diffusion and surface permeation have been observed with many host systems, including the zeolites or zeotypes Ferrierite [9,14], ZSM-5/silicalite-1 [13,15] and SAPO STA-7 $[16,17]$ and the metal-organic framework (MOF) manganese formate [18]. In contrast to these studies, diffusion measurements with $\mathrm{Zn}$ (tbip) revealed a remarkable parallel between diffusivities and surface permeabilities [19] including identical temperature dependencies [20]. In this Letter we develop a model that can explain the experimentally observed interrelation between intracrystalline diffusion and surface permeation. The model assumes total blockage of the vast majority of the pore entrances, with only a few pores directly accessible from the outside. Several examples suggesting almost complete blockage of pores over an entire crystal plane can be found in the literature $[21,22]$.

Synthesis, properties, and potential applications of MOFs of type $\mathrm{Zn}$ (tbip) $\left(\mathrm{H}_{2}\right.$ tbip = 5-tert-butyl isophthalic acid), which are the focus of this study, have been described in Ref. [23]. These materials are distinguished by a high stability, which allowed a totally reproducible performance over a long series of uptake and release measurements, which is a prerequisite for compiling a sufficiently large and reliable set of permeability [19] and diffusivity [8] data for an individual crystal. As illustrated in greater detail in the supplemental material (SM1) [24], the pore space of $\mathrm{Zn}$ (tbip) consists of parallel chains of cages, each of which, under the pressure of the guest molecules in the surrounding atmosphere, can accommodate no more than a single molecule. Accordingly, mass transfer is controlled by one-dimensional diffusion in the channel direction $(x)$, interrupted by the surface barriers, i.e., the finite permeabilities through the surface boundary. The evolution of the intracrystalline concentration profiles during guest uptake or release was analyzed via the diffusion equation (Fick's second law) with the appropriate boundary condition [25].

Figure 1 shows the structural model used to simulate diffusion in $\mathrm{Zn}$ (tbip). The host system is supposed to consist of channels ( $1 d$ pores), in which mass transfer occurs by random molecular jumps (in the $x$ direction) between adjacent segments. Only a small fraction $p_{\text {open }}$ of the channel mouths are open to the surroundings. Filling 


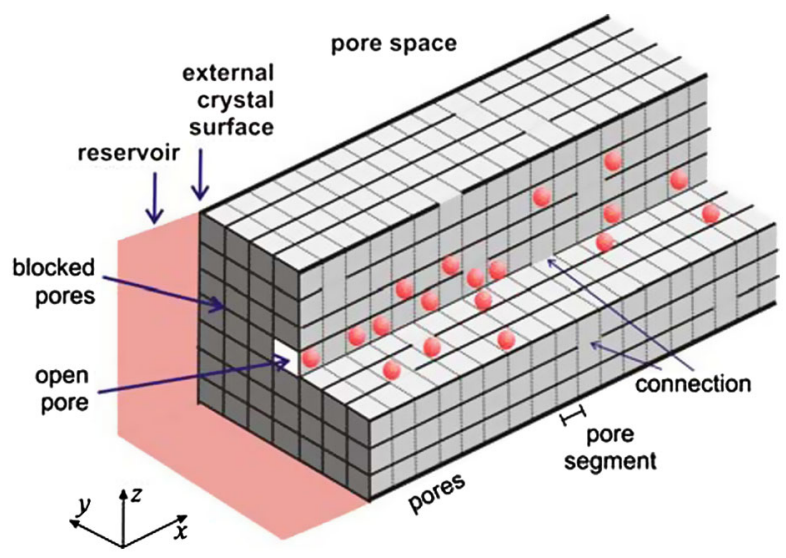

FIG. 1 (color online). The structure model used to simulate mass transfer. The host system consists of $1 d$ channels, where adjacent channels are connected at defect sites occurring with a probability $p_{y}$ and $p_{z}$, respectively. Only a small fraction $p_{\text {open }}$ of the channel mouths are open; i.e., molecules may enter into these channels directly from the surroundings.

of the blocked channels occurs through defects in the channel walls, which occur with the probabilities $p_{y}=p_{z}$. Molecular jumps are "attempted" with equal probability in all six directions. A jump is successful only if it occurs in a direction in which the segment connection is open and if the segment to which the jump is directed is empty. The equilibrium concentration is adjusted by appropriately choosing the encounter rate of molecules from the reservoir onto the crystal surface.

Figure 2 shows the results of such a kinetic Monte Carlo simulation: In the initial state of uptake or release, surface heterogeneity, i.e., the variation between open and blocked entrances, gives rise to heterogeneities in

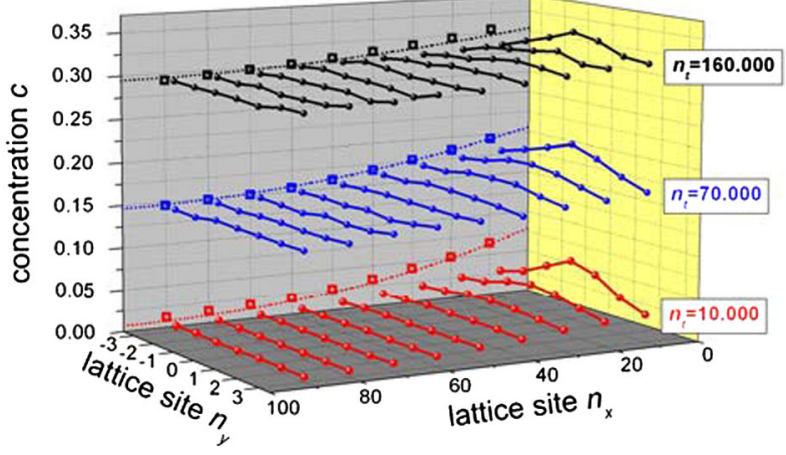

FIG. 2 (color online). Simulated concentration profiles represented as an average in $z$ direction (spheres) and in both $z$ and $y$ direction (squares) after different numbers $n_{t}$ of time steps, together with the solution of the diffusion equation with a finite permeability (dotted lines). The equilibrium concentration is $c_{\text {eq }}=0.5$, the number of lattice sites in $x$ direction is $n_{x}=$ 100 , the probabilities of defects are $p_{y}=p_{z}=0.05$. Out of $n_{y} \times n_{z}=7 \times 7$ sites at the channel entrances $\left(n_{x}=0\right)$ only the central one $\left(n_{y}=n_{z}=0\right)$ is assumed to be permeable. guest concentrations. However, these concentration differences soon disappear with increasing distance from the surface. This is a necessary prerequisite for replacing the effect of permeation through a very small number of open channels by a quasihomogeneous layer of reduced permeability. Mass transfer thus appears to be adequately reflected by an effective-medium approach [26], in complete agreement with the experimental observation and as expected by following the message of the central limit theorem of statistics for displacements notably exceeding the correlation lengths of structural heterogeneities $[27,28]$. It is also for this latter reason that mass transfer perpendicular to the channel direction is not affected by any percolation threshold $[27,29]$. The resulting concentration profiles perpendicular to the surface are found to be well represented by the solution of the transient diffusion equation, based on the intracrystalline diffusivity and a surface permeability. These curves correspond with the experimentally observable concentration profiles.

Surface permeability and diffusivity scale equally with reciprocal time. Their ratio therefore depends exclusively on the geometry of the host system. In the chosen model, host geometry is completely described by the two probabilities $p_{\text {open }}$ and $p_{y}\left(=p_{z}\right)$, yielding a relation of the type

$$
\alpha / D=f\left(p_{\text {open }}, p_{y}=p_{z}\right) / \lambda,
$$

with $\lambda$ denoting the step length, i.e., the distance between adjacent channel sites. Figure 3 displays permeabilitydiffusivity ratios as simulated for different geometrical conditions. Details of the simulations and further examples are provided in SM2 [24]. The simulation data were found empirically to be approximated by the relation

$$
\frac{\alpha \lambda}{D} \approx 0.5 p_{\text {open }} p_{y}\left(1-\frac{p_{y}}{2+4 p_{y}}\right) \frac{5}{1+4 p_{y}}
$$

as illustrated by the dotted lines in Fig. 3. Although, as shown in SM3 [24], the constituents of this approach may be correlated with probability estimates, a rigorous derivation, and an estimate of the range of its applicability beyond the simulations remains a task for the future.
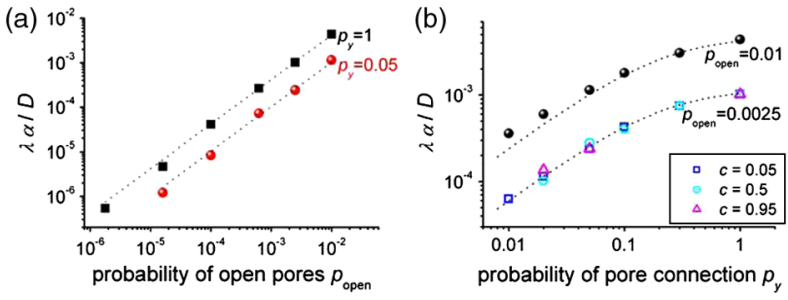

FIG. 3 (color online). Permeability-diffusivity ratio resulting from the simulations, plotted as a function of (a) the fraction of open pores $p_{\text {open }}$ simulated with two values of $p_{y}$ and of (b) the probabilities of pore connections $p_{y}\left(=p_{z}\right)$ simulated with two values of $p_{\text {open }}$. The dotted lines represent the analytical expression provided by Eq. (2). 
The close correlation between the surface permeabilities and the intracrystalline diffusivities [see Eqs. (1) and (2) and Fig. 3] is an immediate consequence of the mechanism to which the surface resistance is attributed, namely, the total blockage of most of the channel entrances. The surface permeability will obviously become smaller, the larger are the "detours" which the guest molecules are forced to take in order to fill the whole channel system. The relative influence of these detours on overall uptake and release rates of guest molecules is determined by the host geometry rather than by the particular mechanisms of molecular transport. SM4 [24] shows the coinciding concentration dependence of the surface permeability and the diffusivity.

In $3 d$ pore networks, i.e., with $p_{y}=p_{z}=1$, for impenetrable surfaces with circular holes of radius $a$ and distance $L$, the permeability-diffusivity ratio is known to be given by $[30,31]$

$$
\alpha / D=2 p^{\prime}{ }_{\text {open }} /(\pi a)
$$

with $p_{\text {open }}$ denoting the fraction of unblocked surface area ("windows"). In SM5 [24], this relation is shown to result as a special case also in our simulations. In addition to the implication that the permeability-diffusivity ratios are merely geometry-dependent quantities, Eq. (3) provides the important information that, for a given fraction $p^{\prime}$ open of open surface area, the permeability and, consequently, the permeability-diffusivity ratio decreases with increasing window diameter. This result indicates, not unexpectedly, a decrease of the "permeation efficiency" of the holes on clustering.

Reference [20] presents an overview of the permeability-diffusivity ratios $\alpha / D$ obtained from the surface permeabilities [19] and intracrystalline diffusivities [8] determined in detailed IRM and IFM experiments with $\mathrm{Zn}$ (tbip), complemented by the very first results of temperature-dependent IFM measurements. In complete agreement with our simulations, for a given crystal these ratios have a well-defined value regardless of whether the measurements were carried out under equilibrium or nonequilibrium conditions (i.e., for both self- and transport diffusivities and the corresponding surface permeabilities) and irrespective of the chosen guest molecules, their concentration and the temperature!

Implying a statistical distribution of the unblocked channel entrances, Eq. (2) allows an estimate of the probability $p_{\text {open. }}$. For this purpose, in SM6 [24] and Ref. [8] the particle passage rate and the exchange probability was determined to $p_{y} \approx 0.05$. Equation $(2)$ in combination with the experimentally determined permeabilitydiffusivity ratio $\alpha \lambda / D \approx 5 \times 10^{-5}$ [20] yields a value of $p_{\text {open }} \approx 5 \times 10^{-4}$ for the probability of finding an unblocked channel entrance. This means that, on the average, only one out of $45 \times 45$ channel entrances is unblocked.
It is important to note that, just as they can occur close to the external surface, such planes of dramatically reduced permeability may also occur statistically distributed throughout the crystal bulk phase [21,32]. When the separation of these planes is much smaller than the crystal diameter (or length), the uptake patterns would become indistinguishable from those under diffusion limitation [33]. The existence of such internal barriers provides a plausible explanation for the remarkable finding that, although differing by orders of magnitude, the diffusivities deduced from "macroscopic" (e.g., uptake) and "microscopic" experiments (with displacements notably smaller than the barrier separations) differ from each other often by one constant factor, leading to the same activation energies [33,34].

For the sake of simplicity, the jump rates through the openings in the blocking plane were assumed to coincide with those in the intracrystalline space. By abandoning this condition of identical jump rates, the relevance of our model is easily seen to be far more general. The data from simulations with different jump rates in the surface and in the pore space are shown in Fig. 4: the difference in the jump rates through the "holes" in the otherwise impenetrable plane and in the pore space is referred to the respective activation energies. The activation energy for jumps through the holes is assumed to exceed those for jumps through the genuine pore space [30,31]. The slopes of the respective Arrhenius plots differ by the ratio between these energies which has been set at 2 for the simulations shown in Fig. 4. Being proportional to the jump rates, also the intracrystalline diffusivity varies with their activation energy. It is important to note that, in the high-temperature range, this is also the activation energy for surface permeation, irrespective of the fact that (except for the highest temperature considered) the jump rates in the bulk exceed those through the holes. This may be rationalized by

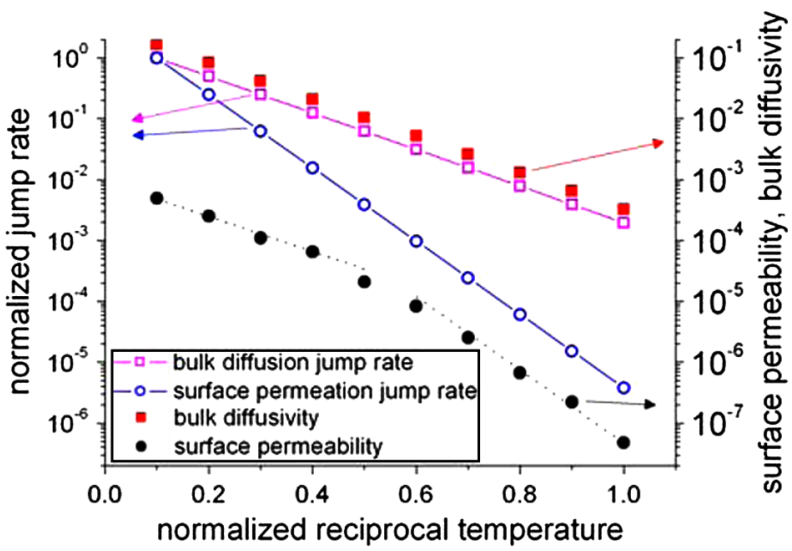

FIG. 4 (color online). Arrhenius plot of the jump rates, surface permeability, and diffusivity. The activation energy for jumps in the surface plane is twice as large as in the bulk crystal. $p_{y}=p_{z}=0.05$ and $p_{\text {open }}=0.03$. 
realizing that for uptake (or pore space filling between adjacent barriers in the crystal interior) a single step through the holes in the barrier must be followed by very many steps within the genuine pore space so that these steps may still remain rate determining. With further decreasing temperature (i.e., with further decreasing ratios of the jump rates) the jumps through the barriers will become increasingly influential so that the rate of (surface) permeation is no longer governed exclusively by the rate of intracrystalline diffusion. Exactly such a situation might lead to the deviations in the trends of surface permeabilities and intracrystalline diffusivities as experimentally observed [9,13-18]. At sufficiently low temperature, the activation energy of permeation will finally coincide with that of the jump rate through the holes in the barrier.

With the crystals of MOF Zn(tbip), we have obviously been able to identify a system which follows the situation in the high-temperature range of Fig. 4. Inversely, those cases where molecular uptake on nanoporous solids is found to reveal activation energies that exceed those observed with microscopically determined intracrystalline diffusivities (e.g., Refs. $[21,33,35]$ ) might be attributed to the situation reflected at medium or lower temperatures, where the permeation rates through the holes in the barriers become relevant.

The experimentally observed correlation between surface permeabilities and intracrystalline diffusivities $[19,20]$ and its reproduction in simulations with impenetrable surface planes and dispersed entrance holes opens a new view of the nature of the surface resistance of nanoporous materials. This view is shown not to be restricted to the rather special situation in the experiments which gave rise to these modeling studies. With increasing transport resistances attributed to the holes, the suggested model is also able to explain existing diffusion and permeation data quite generally. Furthermore, the assumption of a statistical distribution of such resistances over the individual nanoporous particles would yield a self-consistent model that can explain the remarkable similarity in some of the diffusion data obtained from microscopic and macroscopic measuring techniques, despite the large differences in their absolute values [33]. Though being recognized as one of the rate-limiting mechanisms of overall uptake and release, surface resistance has so far remained beyond direct experimental assessment and observation. This situation is changing with the development of microscopic techniques capable of atomistic resolution $[22,36]$. The detection and exploration of microstructural details, which might provide support for our model thus appears to be a promising project for future studies.

We gratefully acknowledge stimulating discussions with Armin Bunde (Giessen, Germany), and Douglas M. Ruthven (Maine, USA), as well as financial support by German Science Foundation, Fonds der Chemischen Industrie and Studienstiftung des Deutschen Volkes.
[1] A. Corma et al., Nature (London) 431, 287 (2004).

[2] C. Serre et al., Science 315, 1828 (2007).

[3] H. M. El-Kaderi et al., Science 316, 268 (2007).

[4] G. Ertl et al., Handbook of Heterogeneous Catalysis (Wiley-VCH, Weinheim, 2008).

[5] H. G. Karge and J. Weitkamp, Adsorption and Diffusion (Springer, Berlin, Heidelberg, 2008), Vol. 7.

[6] J. Kärger, AIChE J. 28, 417 (1982).

[7] J. Kärger et al., Zeolites 6, 146 (1986).

[8] L. Heinke et al., Phys. Rev. Lett. 102, 065901 (2009).

[9] J. Kärger et al., Angew. Chem., Int. Ed. 45, 7846 (2006).

[10] C. Chmelik et al., Chem. Phys. Chem. 10, 2623 (2009).

[11] D. A. Newsome and D. S. Sholl, J. Phys. Chem. B 109, 7237 (2005).

[12] C. Chmelik et al., Chem. Mater. 19, 6012 (2007).

[13] D. Tzoulaki et al., Microporous Mesoporous Mater. 110, 72 (2008).

[14] P. Kortunov et al., J. Phys. Chem. B 110, 23821 (2006).

[15] D. Tzoulaki et al., Angew. Chem., Int. Ed. 47, 3954 (2008).

[16] D. Tzoulaki et al., J. Am. Chem. Soc. 132, 11665 (2010).

[17] L. Heinke et al., Europhys. Lett. 81, 26002 (2008).

[18] P. Kortunov et al., J. Am. Chem. Soc. 129, 8041 (2007).

[19] D. Tzoulaki et al., Angew. Chem., Int. Ed. 48, 3525 (2009).

[20] F. Hibbe, C. Chmelik, L. Heinke, S. Pramanik, J. Li, D. Ruthven, D. Tzoulaki, and J. Kärger, J. Am. Chem. Soc., doi:10.1021/ja108625z (2011).

[21] A. Feldhoff et al., Chem. Phys. Chem. 10, 2429 (2009).

[22] L. I. Meza et al., Phys. Chem. Chem. Phys. 10, 5066 (2008).

[23] L. Pan et al., J. Am. Chem. Soc. 128, 4180 (2006).

[24] See supplemental material at http://link.aps.org/ supplemental/10.1103/PhysRevLett.106.074501 for more details about pore structure, simulations, and data analyses.

[25] L. Heinke and J. Kärger, New J. Phys. 10, 023035 (2008).

[26] C. Tuck, Effective Medium Theory (Oxford University Press, Oxford, 1999).

[27] D. Ben-Avraham and S. Havlin, Diffusion and Reaction in Fractals and Disordered Systems (University Press, Cambridge, 2000), p. 336.

[28] G. H. Weiss, Aspects and Applications of Random Walks (North-Holland, Amsterdam, 1994), p. 361.

[29] A. Bunde and S. Havlin, Fractals and Disordered Systems (Springer, Berlin, 1996), p. 408.

[30] O. K. Dudko, A. M. Berezhkovskii, and G. H. Weiss, J. Chem. Phys. 121, 11283 (2004).

[31] O. K. Dudko, A. M. Berezhkovskii, and G. H. Weiss, J. Phys. Chem. B 109, 21296 (2005).

[32] J. R. Agger et al., J. Am. Chem. Soc. 125, 830 (2003).

[33] D. M. Ruthven, S. Brandani, and M. Eic, in Adsorption and Diffusion, edited by H. G. Karge and J. Weitkamp (Springer, Berlin, Heidelberg, 2008), p. 45.

[34] J. Kärger and D. M. Ruthven, Zeolites 9, 267 (1989).

[35] H. Paoli et al., Microporous Mesoporous Mater. 55, 147 (2002).

[36] L. Gross et al., Science 325, 1110 (2009). 